injection when she presented herself for treatment. Occasionally she used cocain. The Wassermann test was strongly positive.

The patient was given the Towns-Lambert treatment with an initial dose of 1 grain of morphin. She received five infusions in three days.

The first night and the morning of the second day were marked by discomfort with some nausea and vomiting. (The patient was probably started with too small an initial dose of morphin.) The afternoon and evening of the second day were tolerably comfortable, though the patient at times complained of nausea. She managed to sleep the second night between her belladonna capsules. She was nervous and restless the third day, but experienced no acute withdrawal symptoms. She was given 15 grains of chloral and 15 grains of sodium bromid at bedtime, but spent a rather wakeful night. After the third day the patient had only one period of nervousness, this being on the fourth evening. She was given no further hypnotics. She was discharged the sixth day.

CASE 6.-A woman, aged 52, had used morphin for fifteen years, and was using 3 grains daily by hypodermic injection. At times she used cocain. The Wassermann test was strongly positive.

The patient was given the Towns-Lambert treatment with an initial dose of 1 grain of morphin. She received five infusions in three days. She was nervous and restless during the latter half of the second day. She was also nervous on the third day. There was some nausea accompanying the nervousness, but the patient vomited only once or twice. Her discomfort was considerably relieved by the infusions. She was given hypnotics on the third evening, but afterward slept well without them. There was no discomfort after the third day. The patient was discharged on the sixth day.

BROADER ASPECTS OF MORPHINISM

We should bear in mind that 75 per cent. of morphinists have contracted their habit through the careless administration of morphin by physicians; conse- quently they are by-products from the practice of the medical profession. A majority of the remaining 25 per cent. owe their habit largely to the former careless sale of morphin consequent on government negligence.

In regard to the present position of the morphinist : When the Harrison Narcotic Act came into force in March, 1915, it automatically stopped the supply of morphin available to addicts through legitimate channels. It did not stop the morphinist's physiologic need for the drug, however, and it did not provide medical treatment to remove this need. Consequently, while beneficent in its intent and doubtless beneficent in its ultimate and total result, the act imposes unwarranted suffering on existing addicts.

Even the victims of the morphin habit are entitled to a square deal, and it would seem that the least that could be done to alleviate their misery would be to register them and license them to receive their morphin at the hands of a duly authorized physician or government agent. Such a procedure would have the additional advantage of stopping the illicit traffic in morphin.

It would be better to cure these addicts, however, than to license them; and they must be cured in order to be reformed, for their disability is more physical than moral. They were neither cured nor reformed with the enactment of the Harrison act; they were merely designated as the prey of illicit dealers. These dealers create more morphinists; and by their extortion they impel their victims to prostitution and crime. Thus they bring confusion into the reforms originally purposed by the Harrison act.

900 Metropolitan Building.

\section{PNEUMONIA FOLLOWING INFLUENZA (AT CAMP PIKE, ARK.)}

EUGENE L. OPIE, M.D. (ST. Louis)

Lieutenant-Colonel, M. C., U. S. Army

ALlEN W. FREEMAN, M.D. (Columbus, Ohio) Major, M. C., U. S. Army

FRANCIS G. BLAKE, M.D. (Minneapolis) Captain, M. C., U. S. Army

JAMES C. SMALL, M.D. (ChicAgo)

First Lieutenant, M. C., U. S. Army

AND

THOMAS M. RIVERS, M.D. (BALTIMORE)

First Lieutenant, M. C., U. S. Army

CAMP PIKE, LITTLE ROCK, ARK.

The existence of an epidemic of influenza at Camp Pike was recognized when 214 cases of influenza were admitted to the base hospital, September 23. The epidemic was foreshadowed by a steady increase in the number of admissions to the base hospital diagnosed as acute bronchitis. This increase began about September 1, and on September 18 there were fifty admissions with this diagnosis.

Beginning September 23, the number of cases showed a sudden and alarming increase. September 27 , there were 1,037 new cases, and the number continued in the neighborhood of 1,000 a day until October 3 , when the final decline began. During the period from September 20 to October 19, there were 11,899 cases of influenza. Table 1 gives the number of cases of influenza by days from September 1 to October 31, including those cases diagnosed as acute bronchitis during the first part of September, and also the number of cases of pneumonia having onset on each day.

During the two months covered by the table there occurred 12,393 cases of influenza and 1,499 cases of pneumonia. Only two patients died with a diagnosis of uncomplicated influenza (not confirmed by necropsy), while of the patients with pneumonia, 466 died. Of the patients with influenza, therefore, 12.1 per cent. developed pneumonia, and the mortality for pneumonia was 31 per cent. The mortality for the epidemic as a whole was 3.8 per cent. of those attacked by influenza.

The most striking feature of the epidemic was the extremely rapid spread of the infection throughout the camp. Starting September 22 in a regimental area situated in the extreme southwestern corner of the camp, the disease had within four days appeared throughout the camp proper, and four days later appeared in two outlying encampments, situated respectively 1 mile north and 3 miles east of the main camp.

No single organization escaped the infection, and during the thirty days from September 20,23.3 per cent. of the total population of the camp suffered from the disease. The figures given herewith are based on a detailed study of 11,725 cases occurring between September 20 and October 14, and comprise practically the whole of the epidemic.

The disease displayed a striking selective incidence among the men who had been in camp less than one month. During the period from August 20 to October 1 there had arrived in camp 23,216 new men, and during October 553 additional men arrived. Among these 
new men there developed 7,268 cases of influenza, an attack rate of 30.6 per cent. There were in camp, October 1, 28,782 men who had been in camp prior to August 20. Of these, 4,462 developed influenza, an attack rate of 15.5 per cent., or almost exactly half the attack rate of the new men. This disparity between the attack rates of old and new men was true of both white and colored men.

As between white and colored men there were marked and interesting differences in the rates of attack. The total exposed population, including those in camp October 1, and those arriving during October, was 52,551 men, of whom 41,778 were white and 10,773 were colored. The white men developed 10,296 cases of influenza, a rate of 24.6 per cent., while the negroes developed 1,429 cases, a rate of 13.3 per cent.

That this difference in susceptibility is not due to any essential racial immunity is shown by the experience of a draft of 1,954 negroes who arrived in camp between September 23 and 28 . Of these men, 1,860 were transferred between September 27 and September 30 to the negro tent colony at Fort Roots. In this camp at the time of their arrival there were 2,130 negroes, most of whom had been in camp six weeks or longer. There had been no recognized cases of influenza in this camp prior to the arrival of the new draft. September 30, one of the new men developed

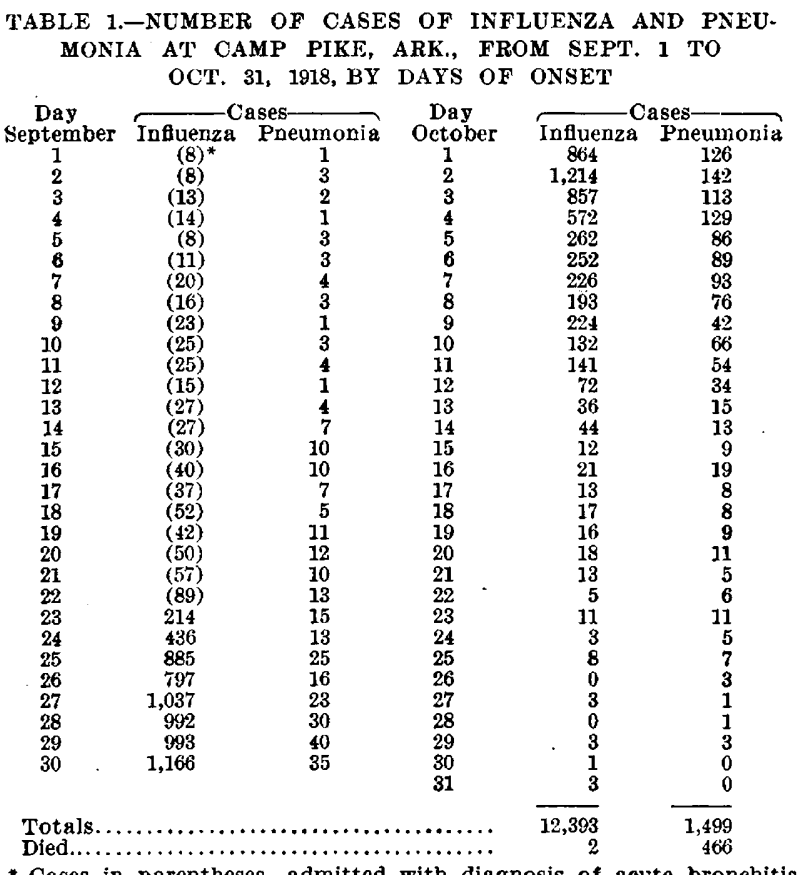

influenza, followed on the next day by forty-seven cases among the new men, and on the next, October 2 , by 462 cases, including a considerable number among the old men. Between September 30 and October 14 there were 972 cases of influenza in the camp, with 107 cases of pneumonia. Of the 972 cases, 810 were in the men of the new draft and 162 in the old men in camp. The rate of incidence for the new men was 43.6 per cent., about as high as that of any organization in Camp Pike, while that of the old men was 7.6 per cent., approximately equal to that of negroes of similar length of service in Camp Pike.

The time of development of pneumonia in relation to influenza is shown in the table. In general, the peak of the curve of pneumonia followed approximately one week after that of influenza, although the highest single day for both coincided. The general incidence of pneumonia followed that of influenza, and the preponderance of new men among the pneumonia cases was even more striking than among the influenza cases. Of the pneumonia patients, 1,006, or 67.5 per cent., were new men, while 62 per cent. of the patients with influenza were new men. The rates of incidence of the two diseases in old and new men are given in Table 2 .

TABLE 2.-INCIDENCE IN OLD MEN AND NEW MEN

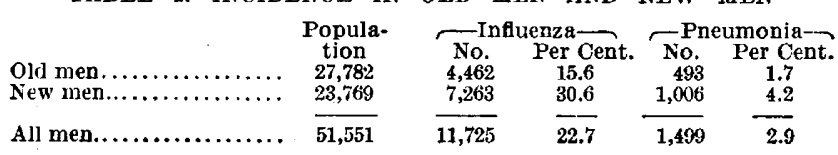

Of the new men with influenza; 13.8 per cent. developed pneumonia, as against 11 per cent. of the old men.

The incidence of pneumonia in cases of influenza was much higher among the negroes than among the whites. Of 10,296 white patients with influenza, 12.7 per cent. developed pneumonia, while among 1,429 negroes with influenza, 283, or 19.8 per cent., developed pneumonia. The rate of fatality from pneumonia was slightly higher in white men than in negroes. Of 1,216 white pneumonia patients, 386 , or 31.7 per cent., died, while of 283 negro pneumonia patients eighty, or 28.2 per cent., died.

September 24, the base hospital contained 2,789 patients; the hospital had been planned to care for 2,009 patients. From September 24 to 30 from 188 to 486 patients were admitted daily to the hospital, so that on September 30 the hospital contained 3,587 patients. October 5 , the number of patients reached the maximum, 4,233. September 24, by order of the camp surgeon the milder cases of influenza were treated in barracks. Every agency for the receiving, the housing and the care of patients throughout the camp was strained by a demand far beyond that for which it had been planned. Patients were crowded in the infirmaries, in the ambulances, in the receiving room of the hospital, in the wards and even in corridors between the wards, where it was essential to shelter them. It was necessary to open and organize in great haste, with-inadequate equipment, wards for the receipt of patients with pneumonia. The medical personnel of the hospital was large at the time; the nursing corps was organized for the care of 2,440 patients ; one quarter of them (sixty-two of 240) were attacked by influenza.

The pressing need of diminishing the overcrowding of the hospital was immediately recognized by. the commanding officer, and through the authorities of the camp, barracks previously used by three replacement -regiments about a quarter of a mile distant were placed at the disposal of the hospital. Patients were moved from the overcrowded hospital into this annex as fast as the facilities available permitted. October 3, 523 patients were transferred to the annex; on the 4 th, 452 ; on the 5 th, thirty-two, and on the 6 th, 387 . In the main hospital, twenty wards for patients with pneumonia were added to the two that already existed. The installation of cubicles and other necessary measures required some time, and during the early period arrangements for the care of patients with pneumonia in some of the new wards were necessarily wholly unsatisfactory. With relief of overcrowding, condi- 
tions rapidly improved. The influence of overcrowding on pneumonia will be discussed later.

\section{INFLUENZA}

The influenza that has occurred here has been characterized by sudden onset with chilliness and sharp elevation of temperature, often from 103 to $105 \mathrm{~F}$. There has been extreme prostration, severe backache, suffusion of the face, and injection of the conjunctivae. Coryza, pharyngitis and tracheitis with a harrassing cough have been almost invariable; epistaxis and slight hemoptysis, frequent. In the majority of cases the temperature has subsided after from two to five days, usually rather abruptly. About one third of the patients have developed purulent bronchitis.

Search has been made for Bacillus infuenzae in a group of twenty-three patients from one to six days after the onset of the disease. From each individual a culture on blood agar ( 5 per cent. horse's blood in meat, infusion agar) has been made (a) from the nose, (b) from the throat and (c) from sputum; and $(d)$ sputum has been injected into the peritoneal cavity of a white mouse.

TABLE 3.-PRESENCE OF B. INFLUENZAE IN TWENTY-THREE

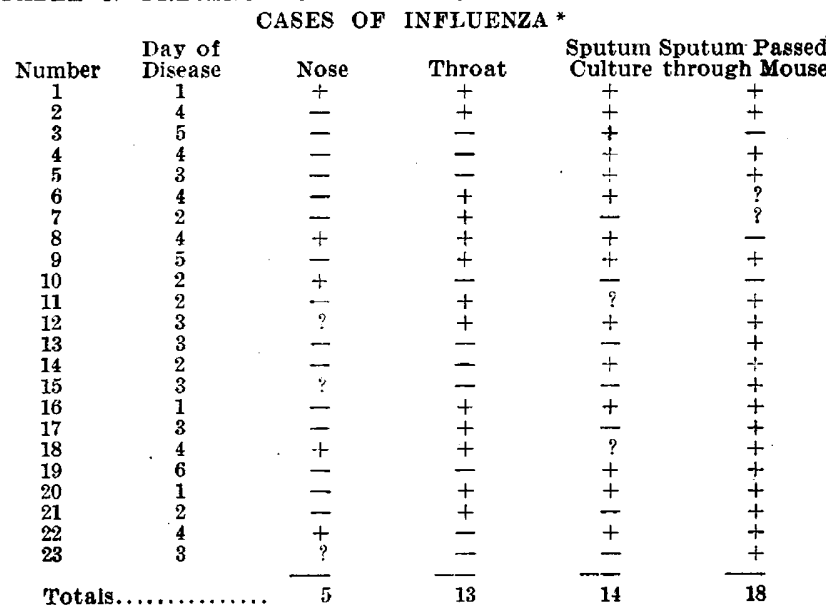

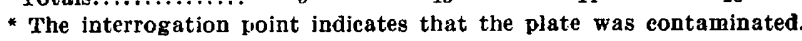

Multiple cultures have demonstrated in some instances in almost pure culture the presence of $B$. infuenzae in all of these cases of early influenza. Passage of sputum through the white mouse has proved the most effective means of demonstrating the organism; cultures from the sputum or throat have been nearly as effective. There is some difficulty in demonstrating the organism in consequence of the minute size of colonies, which may be wholly overlooked by those not familiar with its cultural characters, in the presence of other organisms; for example, in the zone of hemolysis of the hemolytic streptococci the colonies become conspicuous.

\section{BRONCHITIS WITH INFLUENZA}

A considerable number of those attacked by influenza develop bronchitis. Of 103 influenza patients kept under observation, thirty-six had purulent bronchitis. The sputum is profusely mucopurulent and sometimes streaked with blood. In these cases numerous moist râles may be heard. The sputum on direct smear or on culture almost invariably shows $B$. influenzae, often in great numbers.

In attempting to establish the relation of $B$. infuenzae to influenza and its complications, we have borne in mind the observation made at Camp Funston that
$B$. infuenzae was found in the mc uths of 35.1 per cest. of all healthy men examined and was present, in the absence of an epidemic of influenza, in the sputum of a very large proportion of those suffering with bronchitis. Our observations here have shown that the organism is invariably present in the upper respiratory passages of patients with influenza.

\section{PNEUMONIA WITH INFLUENZA}

Pneumonia has occurred in 12.1 per cent. of all influenza patients in this camp, and all pneumonia patients have been treated in the base hospital. Among 103 influenza patients selected for observation from the onset of influenza, four developed clinical evidence of bronchopneumonia and three of lobar pneumonia.

The clinical features of pneumonia following influenza may be best described by division into three groups. It should be borne in mind, however, that the picture has been a complex one and that correct clinical interpretation is not always possible, since many cases do not conform sharply to any one type. These groups are: (1) bronchopneumonia; (2) lobar pneumonia, and (3) lobar pneumonia with purulent bronchitis.

In the first group, bronchopneumonia has usually developed gradually as a sequence to influenza in which purulent bronchitis has occurred, one condition passing into the next without sharp demarcation. In the second group apparent recovery from influenza has occurred, as evidenced by fall of temperature to normal. After from one to three days of normal temperature, typical lobar pneumonia with characteristic rusty sputum has developed suddenly. In the third group, lobar pneumonia has developed in cases of influenza that have been complicated by purulent bronchitis. Such cases have occasionally presented the picture of both lobat pneumonia and bronchopneumonia in the same individual.

Bacteriologic study has disclosed that all these types of pneumonia have been of pneumococcus origin in most instances. As described in greater detail below, some have been further complicated by a superimposed Streptococcus hemolyticus infection.

The bacteriology of the sputum early in the disease has been studied by inoculation of white mice and by direct cultures. The occurrence of pneumococci, $B$. influenzae and Streptococcus hemolyticus in sixty-nine cases of lobar pneumonia has been as follows:

Pneumococcus Type I, nine times (once with Type IV), or 13.1 per cent.; Type II, three times, or 4.3 per cent.; Type II atypical, fifteen times, or 21.7 per cent.; Type III, five times (once with Type IV), or 7.3 per cent; Type IV (alone), thirty-seven times, or 53.6 per cent.

Streptococcus hemolyticus has been found three times, or 4.3 per cent., in all three instances associated with pneumococcus Type IV.

B. influenzae has been found forty-three times, or 62.2 per cent., always associated with pneumococcus (Type I, six times; II, once; II atypical, ten times; III, twice, and IV, twenty-four times).

Pneumococcus Types I and II, which have a predominant part in the production of the pneumonia of civil life, have been present in a relatively small proportion of cases, whereas Type II atypical and Types III and IV are found with 82.6 per cent. of cases.

The bacteriology of the sputum in forty-three cases of bronchopneumonia was as follows:

Pneumococcus Type I, none; Type II, once, or 2.3 per cent.; Type II atypical, three times, or 7 per cent.; Type III, three times, or 7 per cent.; Type IV, thirty times, or 69.8 per cent. 
Streptococcus hemolyticus has. been found six times, or 14 per cent., twice with pneumococcus Type IV and four times with no pneumococci.

$B$. influenzae has been found thirty-eight times, or 88.4 per cent., associated with pneumococci thirty-three times (Type II, once; II atypical, three times; III, three times; IV, twenty-six times); with Streptococcus hemolyticus three times, and alone twice.

Here again pneumococcus Type IV has been found predominant, being present in more than two thirds of the cases. Streptococcus hemolyticus unassociated with pneumococci has been found in 9.3 per cent. of these cases. B. influenzae unaccompanied by pneumococci or hemolytic streptococci was present in two cases; but in view of observations made at necropsy, it is doubtful if this organism alone was responsible for pneumonic consolidation of the lungs.

\section{PATHOLOGY AND BACTERIOLOGY OF PULMONARY LESIONS FOUND AT NECROPSY}

The characters of the group of pneumonias that have occurred in association with the present outbreak of influenza may be defined by the pulmonary lesions found at necropsy, described in relation to the associated bacteria.

Two hundred necropsies have been performed during the outbreak of pneumonia. For the purpose of the present report it has seemed desirable to analyze seventy-nine cases which, occurring with few exceptions in sequence at the height of the outbreak, have been subjected to careful bacteriologic study. Cultures have been made from the heart's blood, from the lung and from a bronchus. When the hemolytic streptococcus has been found in the lung it has usually been demonstrable in the blood. The number of instances in which $B$. influenzae has been obtained in cultures would have been diminished more than half if cultures from the mucosa of the bronchi had not been made.

CHANGES IN THE BRONCHI

Necropsies have shown the presence of purulent bronchitis in a large proportion of those who have died with pneumonia during the course of the present epidemic of influenza. When edema of the lungs is present, the content of the small bronchi is not usually purulent, though the mucosa may be intensely injected. $B$. Influenzae can be grown with few exceptions from the mucopurulent material that wells out of the cut bronchi or from material scraped with a platinum loop from the main branches. In twenty-seven of thirty instances of purulent bronchitis from which cultures were made at necropsy, B. influenzae was found.

When purulent bronchitis is found, the lungs are very voluminous and preserve the shape and size of the thoracic cavity after removal; they show little tendency to collapse, even when cut. Most of the affected lungs, being from men who have come from rural districts, are pale pink and almost wholly free from coal pigment. After section a small droplet of mucopurulent fluid marks the site of each bronchus or bronchiole and doubtless explains the failure of the lung to collapse. The cyanosis of the patients with the disease is doubtless referable in part at least to the same change. The mucosa of the bronchi is intensely injected. Further evidence of severe injury to the bronchi is the frequent occurrence of a zone of hemorrhage about the smaller bronchi, particularly in the lower lobes. At times this zone of hemorrhage gives place to an encircling zone of pneumonic consolidation. Further evidence of profound injury to the bronchial wall is the dilatation that the small bronchi undergo. Bronchiectasis is most conspicuous in the basal part of the lower lobes, and is usually more advanced on the left side than on the right. Small bronchi with no cartilage in their wall may reach a diameter of $0.5 \mathrm{~cm}$. More advanced bronchiectasis has been found in several necropsies performed late in the outbreak.

Among seventy-nine necropsies, selected from the 200 performed, because they have been in sequence and accompanied by careful bacteriologic study, there have been thirty-six instances of lobar pneumonia, nineteen instances of bronchopneumonia, one instance of associated lobar pneumonia and bronchopneumonia, and twenty-three instances of pneumonia with suppuration of lung tissue.

\section{LOBAR PNEUMONIA}

A group of thirty-six cases of pneumonia, taken at necropsy in sequence and studied bacteriologically, gives further knowledge of the bacteriology of the disease as it has occurred here. The restults of this study have been as follows:

Pneumococcus Type I, three times; Type II, none; Type II atypical, five times; Type III, six times; Type IV, nineteen times; total, thirty-three, or 91.7 per cent.

Streptococcus hemolyticus with pneumococci, ten times; with no pneumococci, three times; total, thirteen, or 36.1 per cent.

$B$. influenzae thirty-one times, or 86.1 per cent.

$B$. influenzae has been present with few exceptions. Of pneumococci, which are frequently found in the mouths of healthy men, Type II atypical and Types III and IV are predominant, Type IV occurring in more than half of all cases, whereas Type I has been found only three times in the present series, and Type II has been found in no typical instance of lobar pnewmonia. Hemolytic streptococci have been found in approximately one third of those cases of lobar pnet1monia that have been unassociated with gross evidence of suppuration. It seems probable that the organism has invaded the lung late in the disease, and doubtless the micro-organism in many instances has had an important part in producing the fatal issue. This subject will be considered later when ward infection is discussed. When the hemolytic streptococcus has been unassociated with the pneumococcus it is probable that the latter has disappeared from that part of the lung from which the culture has been taken.

\section{BRONCHOPNEUMONIA}

Two varieties of bronchopneumonia have been observed repeatedly. They do not include all of the instances of bronchopneumonia that occurred: $(a)$ Bronchopneumonia may cause confluent consolidation of large parts of lobes; patches of consolidation, accurately limited to the secondary lobules of the lungs, occur. The larger patches of consolidation are sharply limited by lobule boundaries so that the consolidated tissue on the cut section projects conspicuously above the air-containing lung substance. Even within the large consolidated areas, which are blackish red, red or grayish red and finely granular, the lobules are well defined; but there is no thickening of the interstitial tissue. Two instances of pneumonia due to pneumococcus Type II were of this character. In three other cases the lesion was caused by pneumococcus Type IV. (b) This confluent lobular consolidation is not the pre- 
dominant variety, and disseminated nodules or patches of reddish, gray or yellow consolidation are more commonly seen. The bacteriology of nineteen cases of bronchopneumonia has been as follows:

Pneumococcus Type I, none; Type II, twice; Type II atypical, none; Type III, none; Type IV, ten times; total, twelve, or 70.6 per cent.

Streptococcus hemolyticus with pneumococci, seven times; with no pneumococci, five times; total, twelve, or 70.6 per cent.

$B$, infuenzae, seventeen times, or 89.4 per cent.

Pneumococci doubtless have a predominant part in the production of the'disease, and hemolytic streptococci act as secondary invaders. Nevertheless it is possible that hemolytic streptococci in the presence of influenza may independently invade the lung and produce bronchopneumonia without suppuration.

\section{SUPPURATIVE PNEUMONIA}

Suppuration has occurred in more than one fourth of the cases that have been examined postmortem. Three varieties of suppurative lesion have been found.

(a) Localized abscess formation within a patch of pneumonic consolidation. A patch of consolidation about one abscess is occasionally the only evidence of pneumonia, but more frequently there are multiple patches of bronchopneumonia. The abscess or abscesses are usually situated immediately below the pleura, often separated from the cavity by remains of the membrane no thicker than tissue paper, and the cavity is the site of purulent pleurisy with effusion varying from several hundred up to 1,700 c.c. In all but one of eleven cases of abscess with bronchopneumonia, hemolytic streptococci were found both in the blood of the heart and in the affected lung. In the one exceptional case, pneumococcus Type IV was obtained from the blood; no growth was obtained on the plate inoculated from the lung, and hemolytic streptococci with $B$. influenzae were found in the bronchus. $B$. influenzae was found in the bronchi (six) or in the lung (two) in all but three of these eleven cases. There can be no doubt that the suppurative process due to hemolytic streptococci was in some instances superimposed on pneumonia caused by pneumococci. In one instance, associated pneumococcus Type II was present in blood, lungs and bronchus; in one instance, pneumococcus Type IV was present in blood and bronchus. Of less significance has been the demonstration of associated pneumococci, Type II atypical or Type IV, in the bronchi or lungs (three instances).

(b) Pneumonia with suppuration of the interstitial tissue. In association with bronchopneumonia (three times), with typical lobar pneumonia (twice) or with all defined patches of consolidation (three times), probably best classified as bronchopneumonia, suppuration of the interstitial tissue of the lung has occurred eight times among our cases with completed bacteriologic examination. The interstitial septums appear as conspicuous yellow lines from which purulent fluid may be scraped. The septums are usually swollen to a thickness of about 1 or $2 \mathrm{~mm}$., but wide lines of suppuration $0.5 \mathrm{~cm}$. across are seen. Edematous swelling of septums outside of the consolidated tissue is not infrequently found. These suppurating septums extend up to the pleura, and the overlying lymphatics are often widely distended with fluid. Purulent pleurisy usually with copious effusion up to 1,900 c.c. has been found. In seven of these eight cases, hemolytic streptococci were found both in the heart's blood and in the affected lung. In one instance of lobar pneumonia with interstitial suppuration, we found pneumococcus Type IV with no hemolytic streptococci in the heart's blood and lungs. By unfortunate error, no culture from the affected lung is recorded. The relation of the lesion to associated infection with pneumococci is important. Pneumococcus Type II was found in one instance associated with hemolytic streptococci in the heart's blood, lung and bronchus. In one instance, pneumococcus Type II atypical with $B$. influensae was found in the sputum during life unassociated with hemolytic streptococci, whereas three days later pneumococci were not demonstrable in the lungs or bronchi, and hemolytic streptococci were found in blood, lungs and bronchus. In another case, pneumococcus Type IV and hemolytic streptococci were found in the sputum during life, whereas after death five days later pneumococci had apparently disappeared and hemolytic streptococci were found in the blood and the bronchus, with $B$. infuenzae in the latter situation.

(c) Multiple abscesses clustered about bronchi. In four instances in the group of cases under consideration, suppuration has occurred within bronchopneumonic patches clustered about a medium sized bronchus. These abscess cavities in communication with the bronchus are several millimeters up to 0.5 $\mathrm{cm}$. in diameter, and are surrounded by gray, consolidated tissue. The patch of consolidation studded with abscesses may be scarcely more than $5 \mathrm{~cm}$. across, but a much larger area may be involved. There has been no empyema nor plural effusion in these cases.

The bacteriology of the four cases cited has been as follows :

NECROPSY 280.-Hemolytic streptococci were found in the heart's blood, in consolidated lung tissue and in the bronchus. In the bronchus were found $B$. influenzae and a few staphylococci. Culture from the abscess was contaminated.

NECROPSy 322.-The blood was sterile. Staphylococcus aureus was obtained from consolidated lung; Staphylococcus aureus and pneumococcus Type III were obtained from the abscess.

Necropsy 329.-The blood was sterile. Staphylococcus aureus and pneumococcus Type IV were obtained from the abscess; B. infiuenzae, Staphylococcus aureus and pneumococcus Type IV from the bronchus.

NeCropsy 333.-The blood contained pneumococcus Type II atypical; Staphylococcus aurcus and pneumococcus Type II atypical were obtained from the abscess; Streptococcus hemolyticus from the lung on the opposite side; Staphylococcus aureus, $B$. influenzae and a few hemolytic streptococci from the bronchus.

The foregoing observations indicate that Staphylococcus aureus may be engrafted on a pneumococcus pneumonia and cause suppuration in clustered foci, unaccompanied by empyema.

\section{WARD INFECTION}

Transmission of Streptococcus Pneumonia.-The fatal effect of the necessary overcrowding of this hospital is well shown by a comparison of the death rate from pneumonia in wards, on the one hand, which had long been organized for the care of patients with pneumonia, and in wards, on the other hand, which were opened for patients with pneumonia and immediately overfilled at a time when the hospital was overwhelmed by the epidemic of influenza. 
Ward 3 was provided with cubicles and conducted by medical officers, nurses and enlisted corps men accustomed to the care of patients with pneumonia. The figures in Table 4 show the number of patients in the ward each day from September 6 to October 5 , the number of patients admitted each day and the number of deaths among patients admitted on the corresponding day. The first period of ten days imme-

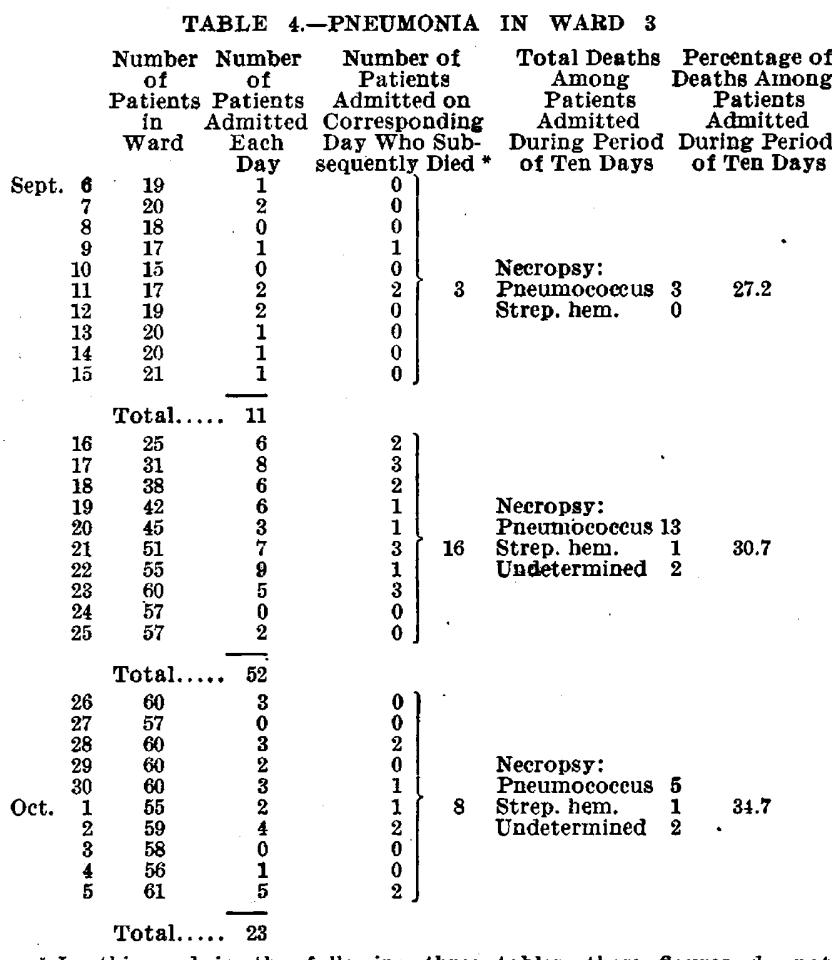

* In this and in the following three tables, these figures do not indicate the number of deaths on the corresponding dates but represent deaths.

diately preceded the present outbreak of pneumonia (Table 1). During the second period of ten days the incidence of pneumonia gradually increased, and during the third period maintained its maximum.

It is noteworthy that the death rate from pneumonia among those admitted during the first ten days was 27.2 per cent. at a time when the ward contained from fifteen to twenty-one patients. It increased during the second ten days, when the ward contained from twenty-five to sixty patients, to 30.7 per cent. With further increase of the ward population to from fiftyfive to sixty-one, the death rate among those admitted during this period rose to 34.7 per cent. If the case of pneumonia due to infection with hemolytic streptococcus which occurred in each of the second and third periods be omitted, the figures for the three periods will be, respectively, $27.2,28.8$ and 30.4 per cent. In spite of the overcrowding of the ward, the introduction of two cases of streptococcus pneumonia under the favorable conditions existing in the ward did not cause an outbreak of the infection.

Ward $8 \mathrm{had}$ long been used for the care of colored patients with pneumonia. Cubicles were in use and precautions were taken against the spread of infection.

During the period from September 1 to September 20 , all new patients with pnetumonia admitted were treated on the porch of the ward, the remaining popuIation of the ward being largely convalescents who were placed inside. During the period from Septem- ber 21 to October 5, when the influenza epidemic was at its height, the ward rapidly filled with active cases of pneumonia and became distinctly crowded. Nevertheless, no outbreak of secondary infection with the hemolytic streptococcus occurred.

Ward 2 was opened, September 28 , during the period in which twenty new wards for pneumonia were organized. From September 26 to October 1, the cubicle system was not in use, the ward was crowded, organization was imperfect, and few precautions were taken to prevent the transfer of infection from one patient to another. October 2 , the cubicle system was introduced, and strict and efficient precautions for the prevention of infection were instituted.

Among patients admitted between September 26 and 28 there was a ward epidemic of streptococcus pneumonia. Nine of the fifty-seven patients admitted to the ward between September 26 and October 1 were shown by examination of the sputum to have pneumococcus pneumonia free from hemolytic streptococci. All died, seven with infection by the hemolytic streptococcus, one with what was presumably an acquired infection with pneumococcus Type III (pneumococcus Type IV was found in the sputum on admission), and one with undetermined cause (no necropsy was held). The sputum of no other patients in this ward was examined on admission.

September 29 (the beginning of the second period), the porch of the ward was put into use, and of twelve patients admitted on this date eleven were put on the

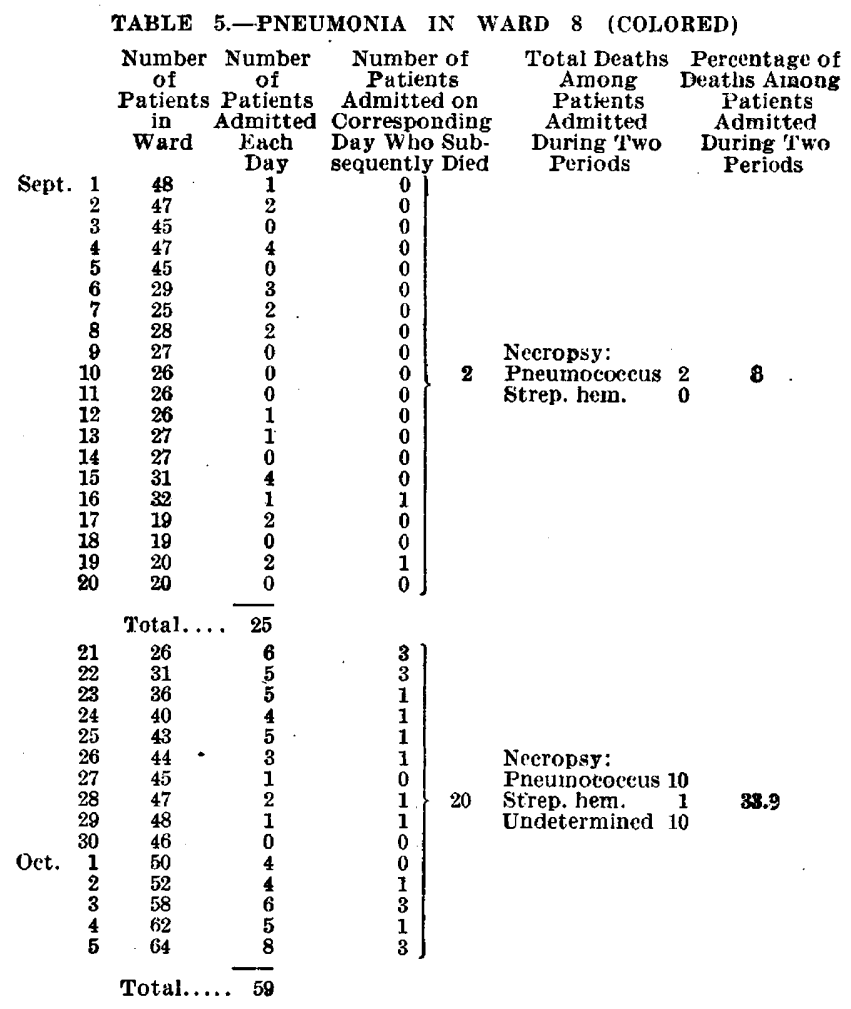

porch. Of these eleven patients, eight recovered and three died, of whom two were transferred to beds within the ward on the day after admission. The use of the porch doubtless explains the diminished mortality of this period.

From October 5 to 7 the ward was closed to further admissions, save six patients transferred from Ward 1 seriously ill; of these all died. After October 7, 
admissions were limited to patients with pneumonia in whom throat swab had demonstrated Streptococcus hemolyticus.

Ward 1 was opened, September 24. During the period from September 24 to October 1, the cubicle system was not in use and there were few precautions to prevent transmission of infection. October 2, the cubicle system was installed, and somewhat insufficient

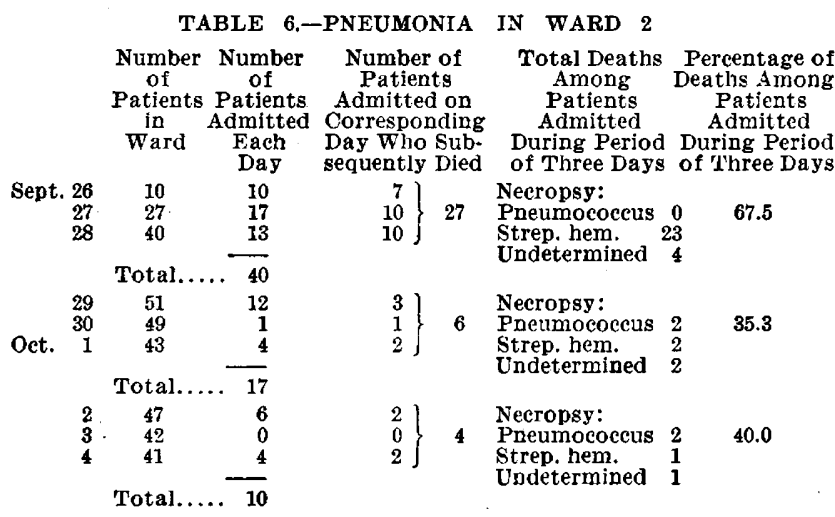

precautions against the spread of contagion were instituted. Beginning October 6 , the ward was closed to further admission. Table 7 is divided into two periods, the first ending September 29, because on September 29 and 30 four patients infected with hemolytic streptococci were admitted to the ward. Deaths that occurred (with no reference to date of admission) prior to September 30 were all due to pneumococcus with one exception, namely, a patient entering the ward on September 26 and dying several hours later.

Hemolytic streptococci introduced into Ward 1 containing patients suffering with pneumonia due, as our experience here has shown, to pneumococci, has produced an epidemic of secondary infection with streptococci. Though the ward was evidently in great danger of infection from September 24 to 29 , as the result of unfavorable conditions, none appears to have occurred; but with the introduction of the micro-

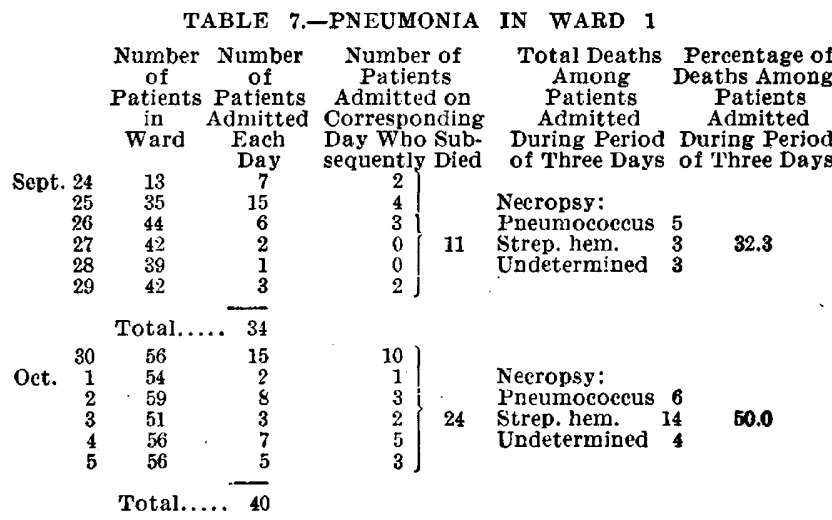

organism, September 29 and 30, widespread dissemination of fatal infection was immediate.

The occurrence of ward infection is well shown by the bacteriologic examination of the heart's blood and lungs at necropsy. Up to October 6, necropsies were performed on nearly all patients dying with pneumonia. The results of bacteriologic examination at necropsy on patients dying in pneumonia wards is given in Table 8 .
The figures demonstrate that routine necropsies with bacteriologic examination of the blood and lungs of every patient that has died with pneumonia furnishes a certain means of recognizing the occurrence of ward infection with hemolytic streptococci. This means of checking the results of bacteriologic examination of the throat and sputum should never be neglected. The recognition of suppurative pneumonia, even when no cultures are made at necropsy, will indicate that pulmonary infection with hemolytic streptococci exists. It is obviously essential to separate pneumonia patients harboring hemolytic streptococci in the mouth from those that are free from this organism; but the existence of hemolytic streptococci in the mouth of patients with pneumonia is not conclusive evidence that the lung has been invaded.

These necropsies, with the accompanying bacteriologic studies, gave us the first conclusive evidence that the pneumonia in this hospital following influenza was subject to secondary invasion with hemolytic streptococci. They showed that Ward 3 had been maintained almost free from pyogenic infection; they forced the conviction that all patients who might subsequently be admitted to Ward 2 would with relatively few excep-

TABLE 8.-BACTERIOLOGIC FINDINGS IN PATIENTS DYING IN PNEUMONIA WARDS *

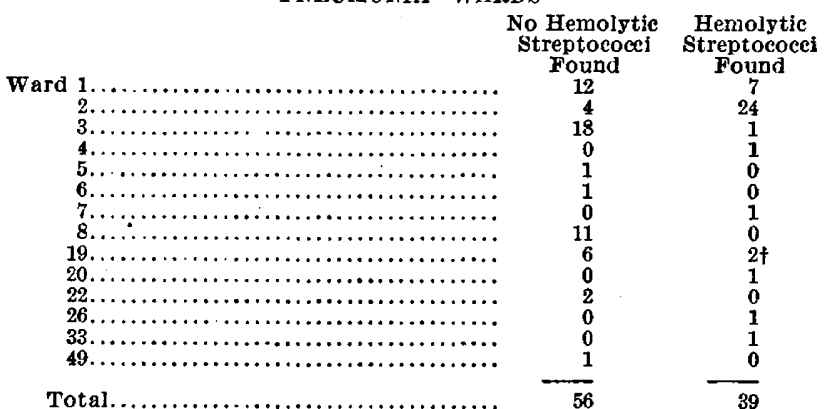

* With the exceptions of Wards $1,2,3$ and 8 , these wards had bee * With the exceptions of
opened less than one week.

+ One of these cases of streptococeus pneumonia was the wardmaster of Ward 2, whose illness began, October 1, during the period of severe tions die with it. The ward was closed. The grave danger to patients entering Ward 1 was recognized, and this ward also was closed to subsequent admissions. The throats of all the 900 patients in the hospital were swabbed as rapidly as accessible facilities permitted, and patients harboring hemolytic streptococci were separated from those that were free from the micro-organism.

Transmission of Pneumococci.-The foregoing observations have demonstrated that streptococcus pneumonia may spread by contagion throughout an entire ward. Subsequent observations have demonstrated that pneumococci may be transmitted in the same way. Opportunity to obtain this evidence has been favored by the predominance of Types IV, III and II atypical among the preumonias occurring here. Among cases of pneumonia of which the type has been determined between September 6 and October 1, only six instances of Type II pneumonia were found. Two of these cases occurred in Ward 3, and there is evidence that the disease was transmitted from these patients to three others in adjacent beds.

Infection with pneumococcus Type II occurred in a patient, Private P., recovering from pneumonia caused by pneumococcus Type IV. This infection with pneumococcus Type. II was apparently acquired from a patient, Private Sw., occupying an adjacent bed. 
Private Sw. was admitted to Ward 3 and placed in Bed 3, September 16, one day after the onset of pneumonia. Pneumococcus Type II was found in his sputum. His crisis occurred, September 21, on the sixth day of his illness. At the time Sw. was admitted, Private P. occupied Bed 5, separated by one bed from Sw.; he had been admitted, September 9, and had bronchopneumonia with signs of consolidation in the left lower lobe. Pneumococcus Type IV was found in his sputum. A crisis occurred, September

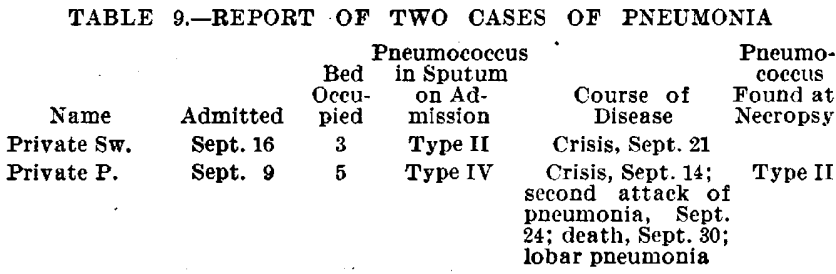

14 , and the temperature remained normal until September 24, save for a sharp rise, September 20 to 21 , lasting eighteen hours. A second attack of pneumonia began, September 24, and death occurred, September 30. Necropsy disclosed the presence of lobar pneumonia with gray hepatization of the greater part of the right lower lobe and of the left lung. Pneumococcus Type II was found in the heart's blood and in the lung, and with $B$. influenzae in the right bronchus. Private $P$. had the opportunity to acquire his second infection from Private Sw.

Infection with pneumococcus Type II occurred in two patients apparently recovering from other types of infection, in one instance caused by pneumococcus Type II atypical, and in another by Type IV. In both instances pneumococcus Type II has apparently been acquired from a third patient occupying an adjacent bed.

Private Sm. was admitted with pneumonia to Ward 3 and placed in Bed 26, September 18. Pneumococcus Type II was found in the sputum. The patient died, September 27, with bronchopneumonia. There were scattered patches of lobular consolidation and diffuse

\begin{tabular}{|c|c|c|c|c|c|}
\hline TABLE & 10.-REPORT & OF & THREE CA & SES OF PNEUMO & ONIA \\
\hline Name & Admitted & $\begin{array}{l}\text { Bed } \\
\text { Occu- } \\
\text { pied }\end{array}$ & $\begin{array}{l}\text { Pneumococcus } \\
\text { in Sputum } \\
\text { on Ad- } \\
\text { mission }\end{array}$ & $\begin{array}{l}\text { Course of } \\
\text { Disease }\end{array}$ & $\begin{array}{l}\text { Pneumo- } \\
\text { coecus } \\
\text { Found at } \\
\text { Necropsy }\end{array}$ \\
\hline Private Sm. & Sept. 18 & 26 & Type II & $\begin{array}{l}\text { Died, Sept. 27; } \\
\text { bronchopneumonia }\end{array}$ & Type II \\
\hline Private $T$. & Sept. 17 & 28 & Atypical II & $\begin{array}{l}\text { Crisis, Sept. 19; } \\
\text { temperature normal } \\
\text { until Sept. 21, when } \\
\text { it rose to 104.4; } \\
\text { death, Sept. 29; } \\
\text { lobar pneumonia }\end{array}$ & $\begin{array}{l}\text { Type II } \\
\text { nal } \\
\vdots \\
;\end{array}$ \\
\hline Private L. & Sept. 16 & 30 & Type IV & $\begin{array}{l}\text { Some improvement } \\
\text { occurred, Sept. } 24 \\
\text { and } 25 \text {, but temper- } \\
\text { ature did not } \\
\text { become normal; } \\
\text { death, Sept. 30; } \\
\text { bronchopneumonia }\end{array}$ & Type II \\
\hline
\end{tabular}

consolidation of the greater part of the right upper lobe. Pneumococcus Type II was found in the heart's blood.

Private T. had been admitted to Ward 3, September 17, with a diagnosis of bronchopneumonia and signs of consolidation in the right lower lobe. Pneumococcus Type II atypical was found in the sputum. Crisis occurred, September 19, and the temperature was normal until 8 p. m., September 21, when there was a sudden rise to 104.4 ; death occurred, September 29. At necropsy there was firm hepatization with yellow color and characters of lobar pneumonia at posterior parts of both lower lobes; but throughout the remainder of the lungs there were small scattered patches of bronchopneumonic consolidation. Pneumococcus Type II was obtained from the heart's blood and lungs; $B$. influenzae was found in the consolidated part of the lung and in the right bronchus. At the time $T$. developed his second attack of pneumonia he occupied Bed 28, separated by one bed from that occupied by Sm., admitted with pneumonia caused by pneumococcus Type II.

Private L. was admitted, September 17, with lobar pneumonia and signs of consolidation in the left lower lobe. Pneimococcus Type IV was isolated from the sputum. There was some improvement, September 24 and 25 , but the temperature never returned to normal and his condition was worse, September 26, when he developed signs of pericarditis. He died, September 30. The left upper lobe was consolidated, rather flabby and had a homogeneous, fleshy appearance. In other parts of the lung there were small scattered patches of bronchopneumonic consolidation and diffuse purulent bronchitis. Pneumococcus Type II with $B$. influenzae was isolated from the right lung, alone from the left lung, and with B. infienzae and Streptococcus viridans from the right bronchus. L., from whose sputum on admission pneumococcus Type IV was obtained, occupied Bed 30, separated by one bed from

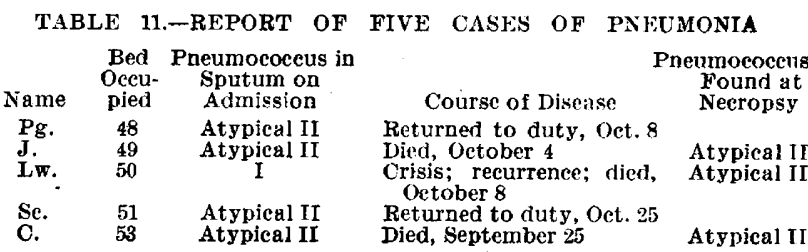

$T$. and by three beds from Sm., both of whom suffered with Type II pneumonia.

The group of cases that has been described is especially significant because these are the only cases of Type II pneumonia save one recognized before October 1. Private P., in Bed 5, doubtless acquired his infection with pneumococcus Type II from Sw. in Bed 3; Private T., in Bed 28, and L. in Bed 30, from $\mathrm{Sm}$. in Bed 26. It is doubtful if the infections of $\mathrm{Sw}$. and $\mathrm{Sm}$. have any relation to each other.

An instance of the transmission of pneumococcus Type II atypical occurred in Ward 8. Privates Sc. and $L$. were admitted to the ward, September 21 , and assigned to Beds 51 and 50 . Privates Pg., J. and C. were admitted to the ward, September 22, and given Beds 48, 49 and 53, respectively. The history of these patients is given briefly in Table 11 .

Private Lw., who became ill one day before his admission, suffered with pneumonia caused by pneumococcus Type I, demonstrated by examination of the sputum on the day following admission. B. influenzae was found. He was in bed adjacent to $\mathrm{J}$. with Type II atypical, who remained beside him until October 4 , and one bed distant from Pg., with Type II atypical, who remained in Bed 48 until October 4, when he was transferred to another part of the ward. On the other side of Lw. was Sc., with Type II atypical, who remained in Bed 51 until October 9, when he was moved to another part of the ward. C., with Type II atypical in Bed 53 died, September 25 . Lw. had frank lobar pneumonia with consolidation of the right lower 
lobe, and crisis occurred, Sept 29 , that is, on the ninth day of illness. The temperature remained normal until October 2, when there was sudden elevation of temperature to 104.2. Death occurred, October 8. Necropsy disclosed lobar pneumonia with gray hepatization of the right upper lobe. The heart's blood and the right lung contained pneumococcus Type II atypical in pure culture. Lw. doubtless acquired his second infection from the group of patients with the same micro-organism surrounding him.

There have been two instances in which, after one type of organism, namely, pneumococcus Type IV, has been found in the sputum by inoculation of white mice and subsequent identification of the pneumococcus, a different type has been demonstrated in the blood or lung at necropsy. Private G. was admitted to Ward 2, September 27, and pneumococcus Type IV and $B$. influenzae were found in his sputum. He died, October 2. There was acute lobar pneumonia with suppuration of the interstitial tissue of the lung and empyema. The heart's blood, lung and bronchus contained pneumococcus Type II;B. influenzae was found in the bronchus. Private Sp. was admitted to Ward 2, September 25. Pneumococcus Type IV was obtained from the sputum. He died, October 3 ; there was acute bronchopneumonia with multiple clustered foci of suppuration, and pneumococcus Type III and Staphylococcus aureus were found in the lung. Even should we assume that pneumococcus Type III in this instance descended into the lungs from the patient's mouth, the case indicates that reinfection of a pneumonic lung with pneumococci is possible.

The foregoing data show that pneumonia of one type does not establish any trustworthy immunity from other types of pneumococci. Patients with one type of pneumococci, notably in the examples cited of Type II, may infect with fatal result patients suffering with or recovering from other types of pneumonic infection. In the absence of streptococcus pneumonia it is essential to maintain in a pneumonia ward precautions that will prevent transmission of infection from one patient to another.

\section{SUMMARY}

During the epidemic of influenza that has been studie', une fourth of those in the camp suffered with the disease; 12.1 per cent. of those attacked with influenza developed pneumonia, and 3.8 per cent. died.

Influenza tended to select newly drafted men, and was twice as common in men who had been in the camp less than one month as in those of longer residence. The incidence of pneumonia among newly drafted men was even higher; 62 per cent. of the cases of influenza were in men in camp less than one month; 67.5 per cent. of cases of pneumonia were in men in camp less than a month.

$B$. influenzae can be demonstrated often in immense number in the nose, throat, or sputum of every patient with influenza, and with few, if any, exceptions in the bronchi of those who have had the purulent bronchitis that follows influenza. The same organism can be found in the throats of some healthy individuals.

Pneumonia follows influenza because the disease renders the air passages susceptible to the invasion of a variety of micro-organisms, among which those commonly found are pneumococci, hemolytic streptococci, and staphylococci.

The profound injury to the air passages produced by influenza is shown by the consequent purulent bronchitis and bronchiolitis preventing collapse of the lung after removal at necropsy, by peribronchial hemorrhage, by dilatation of small bronchi, and ultimately by the formation of multiple bronchiectatic cavities.

Both lobar pneumonia and bronchopneumonia follow influenza, but the disease is somewhat less frequently lobar pneumonia than bronchopneumonia.

Lobar pneumonia following influenza at Camp Pike has been caused in most instances by pneumococci that occur in the mouths of healthy men, namely, by Type IV, 53.6 per cent.; Type III, 7.3 per cent, or Type II atypical, 21.7 per cent., whereas it is seldom caused by Type I, 13.1 per cent, or Type II, 4.3 per cent. Bronchopneumonia has shown similar preponderance of Type IV, 69.8 per cent. It seems probable that Types I and II are much less abundantly distributed among the troops of this camp than in those civil communities where most previous observations have been made.

Among the necropsies that have been performed here, typical lobar pneumonia occurred in about one half of the cases, bronchopneumonia alone in somewhat less than a fourth, and suppurative pneumonia usually superimposed on bronchopneumonia in slightly more than a fourth of the cases.

Three types of suppurative pneumonia occur: (a) Localized abscess formation within a patch of pneumonic consolidation, usually in immediate contact with the pleura and accompanied by empyema. Hemolytic streptococci are found at necropsy in the lung, in the bronchi, in the inflamed pleural cavity, and in the heart's blood. (b) Suppuration of the interstitial tissue of the lung superimposed on bronchopneumonia or occasionally on lobar pneumonia. The suppurative process extends up to the pleural surface, and there is empyema. Hemolytic streptococci are found in the situations named above (under $a$ ). (c) Multiple foci of suppuration clustered about a medium sized bronchus. Empyema has not been found. Staphylococcus aureus has been present in the lesion.

The sequence of events that occurs in many cases of influenza is as follows: $B$. influenzae descends into the bronchi; pneumococci (in this camp usually Type IV) invade the inflamed bronchi, enter the lung, and produce either lobar pneumonia or bronchopneumonia. Hemolytic streptococci may descend and infect the pneumonic lung. It is not improbable that hemolytic streptococci may invade the bronchi previously infected with $B$. influenzae and cause bronchopneumonia in the absence of pneumococci.

When hemolytic streptococci invade the lung either with or without preceding pneumococcus infection, there may be no suppuration of the lung. It is probable that death has occurred before there is opportunity for the formation of abscesses. Streptococcus hemolyticus may pass through a pneumonic lung and appear in the heart's blood although there has been no suppuration. It is not improbable that it may pass through the lung and produce empyema, the lung remaining free from actual suppuration. Lobar pneumonia appears to resist suppuration more effectively than bronchopneumonia; but fatal streptococcus infection is common with both.

Infection with hemolytic streptococci may spread as an epidemic through the pneumonia wards of a hospital. A single patient. with streptococcus pneumonia is a source of grave danger to every patient in 
the same ward. Superimposed infection with hemolytic streptococci increases the mortality of pneumonia so that it may reach from 50 to 100 per cent. of all patients with pneumonia.

Streptococcus pneumonia is introduced into a ward by the admission of a patient with the disease, or doubtless by a patient or attendant harboring hemolytic streptococci in the throat. Transmission of infection is favored by overcrowding, by failure to recognize the disease, by imperfect separation of patients by cubicles and other means, and by imperfect aseptic technic of medical officers, nurses and attendants.

Patients suffering with or recovering from pneumonia caused by one type of pneumococcus may be reinfected with another type of pneumococcus, and fatal pneumonia may result. General conditions that facilitate the transmission of streptococci outlined above favor the occurrence of reinfection with pneumococci.

The history of influenza and pneumonia at Camp Funston ${ }^{1}$ indicates that epidemics of influenza and consequent pneumonia will follow the present epidemics of influenza when new drafts from isolated rural districts are brought into camps that have been attacked by the disease. There seems little reason to doubt that the incidence of pneumonia and the death rate of that which occurs might be greatly diminished by preventing all overcrowding of new recruits in barracks and by providing hospital facilities in considerable excess of the routine demand.

Hospital epidemics of streptococcus pneumonia will be prevented when medical officers have that dread of the disease-comparable to our dread of puerperal fever-which is inevitable when its characters are accurately understood. To promote a more widespread recognition of its nature, the use of a readily understood designation, such as streptococcus pneumonia, should be encouraged.

Streptococcus pneumonia is a preventable disease: (a) The technic of a pneumonia ward must be comparable to that of the best maternity hospitals, with added precautions to prevent patients from disseminating by coughing or other means ejected material containing streptococci, pneumococci or other microorganisms. (b) The throats of all patients with pneumonia should be swabbed before they are admitted to a ward set aside for the treatment of pneumonia in order to determine if they harbor hemolytic streptococci (demonstration of hemolytic streptococcus in the throat does not prove that the lung is invaded). (c) Patients with pneumonia harboring hemolytic streptococci should be kept in a separate ward into which none free from these micro-organisms are admitted. (d) When streptococcus pneumonia makes its appearance in a pneumonia ward, the ward should be closed to further admissions. It would doubtless be closed if diphtheria or scarlet fever had appeared and the danger from streptococcus pneumonia is greater. (e) Necropsies should be performed on every patient dying with pneumonia, and appropriate bacteriologic cultures should be made from the heart's blood and from the lungs as a means of determining the presence or absence of streptococcus pneumonia and to serve as an index of the efficiency of the precautions in force. 1. Opie, E. L.; Freeman, A. W.; Blake, F. G.; Small, J. C., and
Rivers, T. M.: Pneumonia at Camp Funston, J. A. M. A. T2, 108 (Jan. 11) 1919 .

\section{FEIGNED AMNESIA AS A DEFENSE REACTION *}

GEORGE E. PRICE, M.D. (Philadelphia)

Major, M. C., U. S. Army; Consultant in Neuropsychiatry, District of Paris

$$
\text { AND }
$$

WILIIAM B. TERHUNE, M.D. (New ORLEANS) First Lieutenant, M. C., U. S. Army

$$
\text { A. E. F., FRANCE }
$$

The examination of soldiers under arrest at the office of the provost marshal of Paris has revealed a number of individuals feigning loss of memory for the purpose of escaping punishment. This form of malingering was employed as a last resort when the culprits were without other excuse. The charge under which they were held was in every instance that of being absent from their unit without permission, and in no case was amnesia alleged to escape duty at the front.

It was interesting to note that all of these men were native Americans. None presented any evidence of mental deficiency, the majority being somewhat above the average of intelligence. One was a captain, two were first lieutenants, and the others were privates. Such civil occupations were represented as machinist, newspaper reporter, court stenographer, telegrapher and student.

The condition observed was characterized by an alleged absolute blank in the continuity of memory, corresponding to the period of time during which the individual thought it necessary to be excused from his actions. This period varied according to the astuteness of the soldier. Some deened it wise to remember nothing of their past life, others limited it to incidents occurring during the past few years, while the majority claimed an amnesia corresponding to the period of their misdemeanor.

A typical statement would be somewhat like this: "The military police picked me up in the street because I had no papers. I do not know how I got to Paris, how long I have been here, or what I have done. The first thing that I remember was this morning."

\section{REPORT OF CASES}

The subjoined reports are abstracted from the records:

Case 1.-A private stated that he had arrived in Paris two nights before, he did not know how. He went to a militar policeman and gave himself up. He did not know what his organization was or when he enlisted, but he was not drafted. His home was in California, he did not know where. He was not married. He came to France in cold weather, he did not know when. He landed in France, he did not know at what port. He did not know the name of the boat he crossed on; it had one smokestack. He thought he had been a teamster in France. He did not know what he did in civil life. He had been at the front.

The patient denied memory for recent and past events. His attention was good. There was no disorientation nor confusion. He answered questions promptly. He was able to answer problems involving multiplication and division promptly and accurately.

Physical examination was negative.

CAse 2.-A private was not sure of his name. He found an envelop in his pocket and he imagined it was addressed to him. This letter was from California. He said that he thought California was in the United States, but he could not say where the United States was. He did not know

\footnotetext{
* From American Red Cross Military Hospital No. 1.
} 